\title{
Editorial:
}

\section{Complex system and intelligent control: theories and applications}

\author{
Jie CHEN ${ }^{\dagger 1,2}$, Ben M. CHEN ${ }^{3,4}$, Jian SUN ${ }^{1}$ \\ ${ }^{1}$ School of Automation, Beijing Institute of Technology, Beijing 100081, China \\ ${ }^{2}$ Tongji University, Shanghai 200092, China \\ ${ }^{3}$ Department of Mechanical and Automation Engineering, Chinese University of Hong Kong, Hong Kong, China \\ ${ }^{4}$ Department of Electrical and Computer Engineering, National University of Singapore, Singapore \\ ${ }^{\dagger}$ E-mail: chenjie@bit.edu.cn
}

https://doi.org/10.1631/FITEE.1910000

Complex systems are the systems that consist of a great many diverse and autonomous but interacting and interdependent components whose aggregate behaviors are nonlinear. As phased by Aristotle, "the whole is more than the sum of its parts;" properties of complex systems are not a simple summation of their individual parts.

Complex systems are widespread. Typical examples of complex systems can be found in the human brain, flocking formation of migrating birds, power grid, transportation systems, autonomous vehicles, social networks, and communication networks.

Complex systems have some distinct properties, such as highly nonlinear dynamics, emergence, adaptation, and self-organization, which are difficult to model precisely. Such properties lead to difficulties in understanding the behaviors of complex systems, to model them accurately, to control them, and to make them work in a specific way we desire.

There is no generally agreed definition of intelligent control. Generally speaking, intelligent control is a class of control methods that use artificial intelligence techniques, such as fuzzy logic, neural networks, evolutionary computation, machine learning,

\footnotetext{
ॠ Corresponding author

(c) Zhejiang University and Springer-Verlag GmbH Germany, part of Springer Nature 2019
}

and swarming intelligence. Intelligent control tries to borrow ideas from the sciences of physics, mathematics, biology, neuroscience, and others to develop a control system to manage a complex process in an uncertain environment. Intelligent control is a kind of interdisciplinary new technology, which integrates the knowledges of control theory, computer science, artificial intelligence, information theory, and other areas. Intelligent control has been shown to be effective in controlling complex systems where traditional control methods can hardly perform well.

In this context, the Chinese Academy of Engineering (CAE) organized a special issue on complex system and intelligent control in Frontiers of Information Technology and Electronic Engineering. This special issue aims to promote research on complex systems and intelligent control and reflect the most recent advances, with emphasis on both theories and applications. After rigorous review process, 11 papers by researchers worldwide have been selected for this special issue, including one survey paper and 10 research papers.

Electric power grid as a typical example of complex systems has brought a great change in our daily lives. An accurate, fast, and robust power system state estimation is undoubtedly significant. Gang WANG et al. gave a comprehensive review on some 
of the recent advances in power system state estimation with an emphasis on the solvers that can efficiently obtain optimal or near-optimal solutions to nonconvex state estimation tasks. Some directions for future research were also discussed.

Motion coverage is an important real-world application for mobile robots. To deal with the multirobot coverage motion planning problem, Guan-qiang GAO and Bin XIN proposed an auction-based spanning tree coverage (A-STC) algorithm. Compared with existing methods, this algorithm is effective, time-efficient, and complete, especially in large complex configuration spaces.

A snake robot is a biomorphic robot to imitate snake's morphology. To make a snake robot as agile as a biological snake, locomotion generation and body motion control are two important issues. Wenjuan OUYANG et al. proposed a biomimetic control approach for steering a snake robot. The proposed method includes an artificial central pattern generator, a cerebellar model articulation controller, and a proportional-derivative controller. Their simulations and experiments demonstrated good performance and adaptability of the method.

Ning-shi YAO et al. presented an autonomous robotic blimp equipped with only one monocular camera, named Georgia Tech Miniature Autonomous Blimp (GT-MAB). It was designed to support human robot interaction experiments in an indoor space. The blimp is capable of effectively detecting the face of a human subject, following the human, and recognizing hand gestures by employing a deep neural network based algorithm. Their experimental results demonstrated that GT-MAB has reliable human-detection and human-following capabilities.

Execution control is a critical task of robot architecture design, and has important influence on the quality of the final system. Martin MOLINA et al. presented a general method for execution control. The proposed method was integrated in the Aerostack open-source framework. Their experiments demonstrated the validity, feasibility, and effectiveness of the proposed method.

In recent years, multi-agent systems have re- ceived much attention since their wide applications in mobile sensor networks, formation of unmanned flight vehicles, smart grids, etc. Formation control is an important topic in cooperative control for multiagent systems. Mao-peng RAN et al. considered the time-varying formation tracking problem for multiagent systems subject to unknown nonlinear dynamics and external disturbances. An extended state observer (ESO) was designed to estimate the total uncertainty of the system. An ESO-based time-varying formation tracking protocol was proposed. Effectiveness of the theoretical results was demonstrated by application to the target enclosing problem of a group of unmanned aerial vehicles (UAVs). Mao-bin LU and Lu LIU studied the leader-following consensus problem of a class of heterogeneous second-order nonlinear multi-agent systems subject to disturbances. A class of distributed control laws that depend on the relative state of the system were proposed to solve the problem.

In recent years, UAVs have been widely applied in many fields, such as reconnaissance, search and rescue, surveillance, environment monitoring, largeaccident investigation, parcel delivery, and agriculture service. Among all the UAVs, the quadrotor platform is the most popular one. Control and navigation of quadrotor UAV has received much attention. Yu-jiang ZHONG et al. presented a reliable active fault-tolerant tracking control method for a quadrotor UAV with model uncertainties and actuator faults. A radial basis function neural network was introduced to estimate the model uncertainties online, modify the reference model adaptively, and incorporate it into the model reference adaptive control scheme. To deal with actuator faults, a fault detection and diagnosis estimator and a fault compensation term were introduced into the control law. Shu-peng LAI et al. proposed a computationally efficient safe flying corridor navigation method for a quadrotor UAV. This method can generate a jerk-limited trajectory within a safe corridor. Unlike the existing methods, the proposed method can generate a smooth non-stop trajectory with safety guarantee but does not require the vehicle stop at each intersected point of the connected boxes. 
A flywheel suspended on active magnetic bearings as a kind of complex system has been found to have many applications in energy storage devices. However, the controller design problem for a flywheel system is still challenging. Xujun LYU et al. applied the characteristic model based all-coefficient adaptive control method to active magnetic bearings suspended flywheel systems. An extensive simulation showed that the characteristic model based allcoefficient adaptive control method has considerable robustness with respect to plant uncertainties, external disturbances, and time delay.

Ze-zhi TANG et al. investigated the disturbance rejection problem of active magnetic bearing systems. A disturbance observer based iterative learning control strategy that combines the extended state observer with a classic iterative learning control law was proposed. Their simulation and comparison confirmed that the proposed method can yield good tracking performance.

The papers included in this special issue cover a broad spectrum of current research topics on complex systems and intelligent control, including formation control of complex multi-agent systems, control and navigation of a quadrotor UAV, robust control of robots, interaction between human and robot, control of systems with model uncertainties and disturbances. We hope that this special issue will benefit the researchers in these fields and foster research on complex systems and intelligent control.

This special issue would not be possible without the support of many people, including the authors and reviewers. We are indebted to the editorial staff of the journal for their enormous assistances, and to the Editors-in-Chief, Profs. Yun-he PAN and Xi-cheng LU, for this great opportunity.

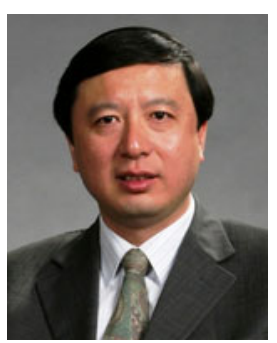

Prof. Jie CHEN received BS, MS, and $\mathrm{PhD}$ degrees in control theory and control engineering from the Beijing Institute of Technology, Beijing, China, in 1986, 1993, and 2001, respectively. He is currently the President of Tongji University, China. He is an Academician of the Chinese Academy of Engineering and IEEE Fellow. His current research interests include intelligent control and decision in complex systems, multi-agent systems, and optimization methods.

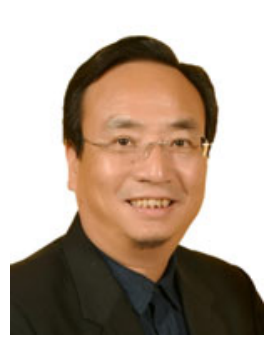

Prof. Ben M. CHEN received the BS degree in computer science from Xiamen University, China in 1983, MS degree in electrical engineering from Gonzaga University, USA in 1988, and $\mathrm{PhD}$ degree in electrical and computer engineering from Washington State University, USA in 1991. He is currently a professor in the Department of Mechanical and Automation Engineering at Chinese University of Hong Kong, and a professor in the Department of Electrical and Computer Engineering, National University of Singapore. His current research interests include unmanned systems, robust control, and control applications. He is an IEEE Fellow.

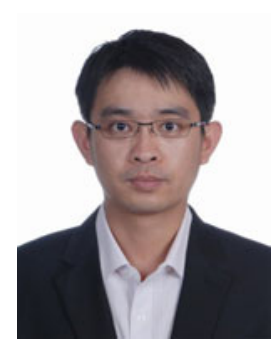

Prof. Jian SUN received the BS degree from the Department of Automation and Electric Engineering at Jilin Institute of Technology, China in 2001, the MS degree from Changchun Institute of Optics, Fine Mechanics and Physics, Chinese Academy of Sciences (CAS), China in 2004, and the PhD degree from Institute of Automation, CAS, China in 2007. He is currently a professor in School of Automation at Beijing Institute of Technology, China. His current research interests include networked control systems, timedelay systems, and security of cyber-physical systems. 